\title{
Exceptionally Elastic Single-Component Pharmaceutical Crystals
}

\author{
Kunlin Wang, ${ }^{\dagger}$ Manish Kumar Mishra, ${ }^{\dagger}$ and Changquan Calvin Sun*(0) \\ Pharmaceutical Materials Science and Engineering Laboratory, Department of Pharmaceutics, College of Pharmacy, University of
} Minnesota, 9-127B Weaver-Densford Hall, 308 Harvard Street S.E., Minneapolis, Minnesota 55455, United States

Supporting Information

\begin{abstract}
We report here the first elastically bendable singlecomponent pharmaceutical crystal, celecoxib. Interlocked molecular packing without the slip plane and the presence of an isotropic hydrogen bond network are major structural features responsible for both the exceptional elastic flexibility and high stiffness of the celecoxib crystal as revealed by bending and nanomechanical studies. The molecular model of the exceptional elasticity is rationalized by the inhomogeneous spatial separations of molecules in the bent crystal, which is further confirmed by micro-Raman spectroscopy. The celecoxib crystal, exhibiting both therapeutic effects and elastic mechanical behavior, could be used to manufacture functional microdevices with novel medical applications.
\end{abstract}

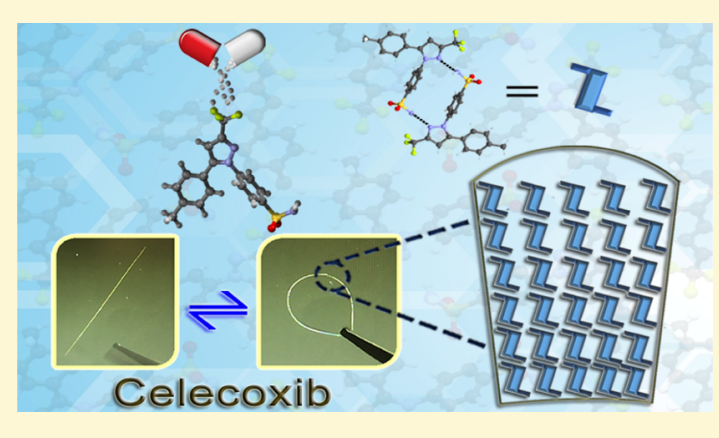

Mira olecular crystals with exceptional mechanical properties have gained prominent attention because of the wide range of potential applications, such as pharmaceuticals, explosives, flexible optoelectronics, bioinspired natural fibers, food, and fine chemicals. ${ }^{1-14}$ Mechanical behavior of molecular crystals depends on the types of the atoms, ions or molecules, molecular arrangement, and strength of intermolecular interactions. ${ }^{1-6,15}$ Property engineering in molecular crystals is the main objective of the third-generation crystal engineering, which is accomplished via the modulation of the underlying structure. ${ }^{2,4}$ Crystal engineering is also widely used in designing active pharmaceutical ingredients (APIs) with optimum physicochemical properties, such as solubility, dissolution rate, bioavailability, and mechanical properties. ${ }^{2,4,6,16-18}$ Understanding the mechanical behavior of pharmaceutical crystals in the context of crystal packing, slip planes, and crystal defects is of significant importance both scientifically and practically. ${ }^{16-23}$ For instance, the success in the processes of milling and tableting of an API depends, to a large extent, on its mechanical properties. ${ }^{6}$ A key to an effective design of pharmaceutical crystals with optimum mechanical properties is the establishment of an appropriate structuremechanical property relationship.

Mechanical plasticity in most molecular crystals can be rationalized based on crystal packing anisotropy and the presence of slip systems. ${ }^{1-6}$ Hand-twisted helical crystals have also been recently designed by introducing two-dimensional plasticity with a fair degree of isotropic crystal packing. ${ }^{2}$ In contrast, elasticity predominately depends on the isotropy of both crystal packing and strength of intermolecular interactions. ${ }^{1-6}$ Examples of elastic behavior of molecular crystals and cocrystals have been reported and rationalized. $^{4,5,14,15,24-33}$ Elastic crystals of drugs with therapeutic characteristics could be a class of materials with novel mechanopharmaceutical applications. ${ }^{1-6,34-36}$ For example, a pharmaceutical crystal with combined elasticity and optical fluorescent properties could be exploited for medical and diagnostic imaging in biomedical applications. ${ }^{34,35}$ Despite the appealing aspects of elastically bending crystals with biopharmaceutical activities, their design is extremely difficult due to a lack of clear understanding of the structure-elasticity relationship at the molecular level.

So far, the known elastic pharmaceutical crystals are all multicomponent, for example, cocrystal, solvate, and salt. $^{22,36,37}$ The first reported elastic molecular crystal was a caffeine cocrystal solvate. ${ }^{24}$ Subsequently, an elastic biocrystal of clofazimine hydrochloride salt was discovered. ${ }^{37}$ In both cases, the solvent and chloride anion play an integral role in the structural stability and elasticity during bending. Recently, a cocrystal of 4,4'-azopyridine and probenecid, a uricosuric agent, has been shown to exhibit unique elastic behavior under external stimuli of heat, light, or mechanical load. ${ }^{38}$ However, an elastic pharmaceutical single-component crystal remains to be discovered. Herein, we report the first elastic crystal of a blockbuster drug, celecoxib Form III (CEL, Figure 1). CEL is a COX-2 selective nonsteroidal anti-inflammatory drug for treating pain and inflammation associated with osteoarthritis or rheumatoid arthritis, and other acute pain in adults. ${ }^{39,40}$ Although no extraneous molecules are present in the crystal lattice, CEL still exhibits surprisingly elastic bending behavior. We conducted bending and indentation experiments to quantify the elastic properties of the CEL crystal and identified responsible features in the crystal structure and key intermolecular interactions.

Acicular-shaped CEL crystals, 3-5 $\mathrm{mm}$ in length and 0.05$0.1 \mathrm{~mm}$ in width, were obtained by slow evaporation of a

\footnotetext{
Received: January 5, 2019

Revised: February 15, 2019

Published: February 19, 2019
} 


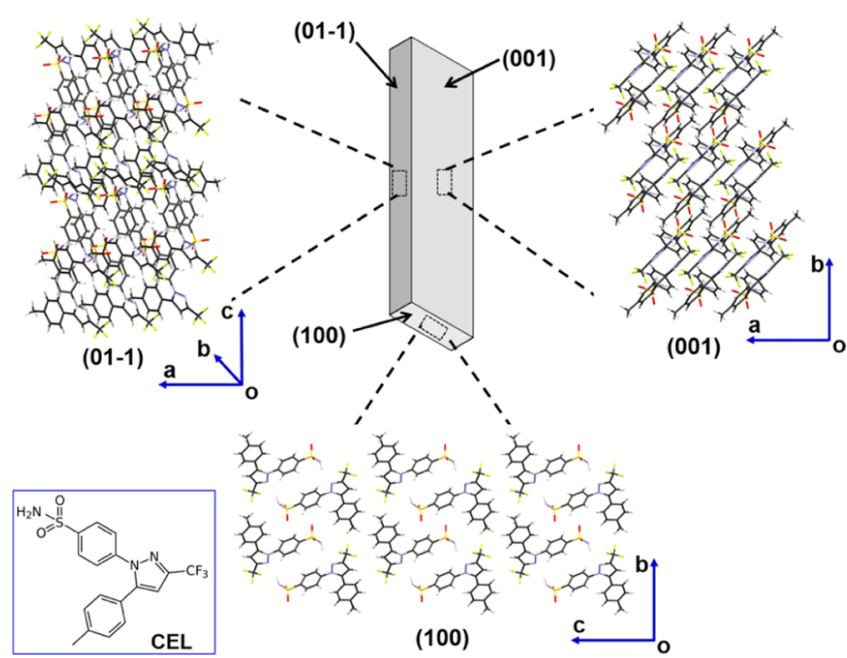

Figure 1. Molecular packing in CEL viewed into (01-1), (001), and (100) faces.

methanol solution at room temperature (see S1). The previously reported crystal structure of CEL was of insufficient quality $(R$ factor $=8.8 \%) .{ }^{39}$ For accurate structural analysis, we solved the CEL crystal structure with a higher quality $(R$ factor $=4 \%)(\mathrm{S} 2$ and Table S1). The two dominant faces of CEL crystals were $(001) /(00-1)$ and $(01-1) /(0-11)$, and the end faces of the crystals were $(100) /(-100)$, identified from a faceindexing experiment using single-crystal X-ray diffraction (SCXRD) (Figures 1 and S1).

A crystal structure analysis of CEL shows that the 4methylphenyl and trifluoromethyl- $1 \mathrm{H}$-pyrazol rings are approximately in plane $\left(18^{\circ}\right)$ and both are approximately perpendicular $\left(89.56^{\circ}\right.$ and $83.78^{\circ}$, respectively) to the benzenesulfonamide ring (Figure $\mathrm{S} 2$ ). The $-\mathrm{NH}_{2}$ group of benzenesulfonamide of each CEL molecule is bonded with $\mathrm{N}$ of the pyrazol ring to another CEL molecule $(\mathrm{N}-\mathrm{H} \cdots \mathrm{N}, D, d$, $\theta$ : $\left.3.038 \AA, 2.19 \AA, 159^{\circ}\right)$ to form a hydrogen-bonded dimer (Figure $2 \mathrm{a}$ ). The dimer extends along $a$-axis to form a onedimensional (1D) chain fortified by $\mathrm{N}-\mathrm{H} \cdots \mathrm{O}$ (2.922 $\AA$, 2.09 $\left.\AA, 166^{\circ}\right), \mathrm{C}-\mathrm{H} \cdots \mathrm{N}\left(3.517 \AA, 2.740 \AA, 139.79^{\circ}\right), \mathrm{C}-\mathrm{H} \cdots \pi$ (3.869 $\left.\AA, 2.966 \AA, 159.98^{\circ}\right)$, and $\mathrm{C}-\mathrm{H} \cdots \pi(3.805 \AA$, $2.976 \AA$, $143.97^{\circ}$ ) hydrogen bonds (Figures $2 \mathrm{~b}$ and S3a). Neighboring $1 \mathrm{D}$ chains are connected by various weak intermolecular interactions, that is, the $\mathrm{C}-\mathrm{H} \cdots \mathrm{F}$ dimer $(3.516 \AA$, $2.59 \AA$, $\left.165.2^{\circ}\right), \mathrm{C}-\mathrm{H} \cdots \mathrm{F}$ linear $\left(3.578 \AA, 2.696 \AA, 149.95^{\circ}\right)$, and C$\mathrm{H} \cdots \pi$ dimer $\left(3.936 \AA\right.$, $\left.2.971 \AA, 168.4^{\circ}\right)$. CEL dimers from neighboring $1 \mathrm{D}$ chains are offset by $4.896 \AA$ along the direction of chain. The connection of dimers via the $\mathrm{C}-\mathrm{H} \cdots \pi$ dimer (3.936 $\AA, 2.971 \AA, 168.4^{\circ}$ ) hydrogen bond leads to corrugated layers parallel to the (100) plane (Figure S3b). Each corrugated layer is connected by the bifurcated $\mathrm{C}-\mathrm{H} \cdots \mathrm{O}$

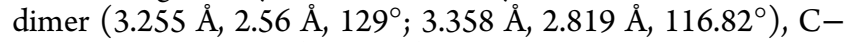
$\mathrm{H} \cdots \mathrm{F}$ dimer $\left(3.516 \AA\right.$, $\left.2.59 \AA, 165.2^{\circ}\right)$, and $\mathrm{C}-\mathrm{H} \cdots \mathrm{F}$ linear (3.578 $\left.\AA, 2.696 \AA, 149.95^{\circ}\right)$ to form interlocked packing along the (001) plane (Figures 2 and S3). Overall, the structural features of CEL satisfy the essential criteria for elastic crystals with a specific bendable face: (1) interlocked isotropic packing without any slip planes, which prevents long-range molecular movement to avoid plastic deformation during flexing; and (2) presence of multiple dispersive interactions in orthogonal directions, which leads to "structural buffering" to allow (a)
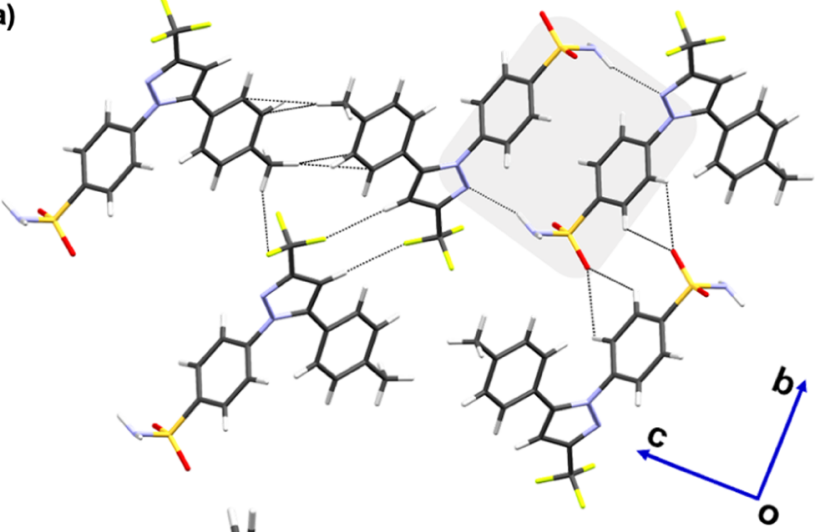

(b)

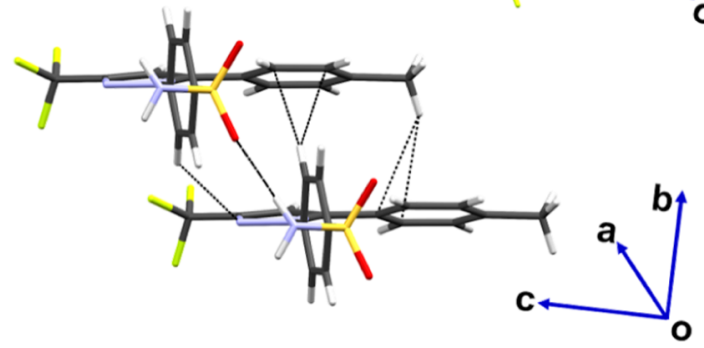

Figure 2. Intermolecular interaction pattern from two different orientations (a) and (b). Interaction within the dimer is highlighted in the gray color.

reversibly stretched intermolecular interactions during elastic bending. ${ }^{24,25,33}$

An acicular CEL crystal could be easily and repeatedly bent (Figure $3 a-i$, see Video S1), when a load was applied to the

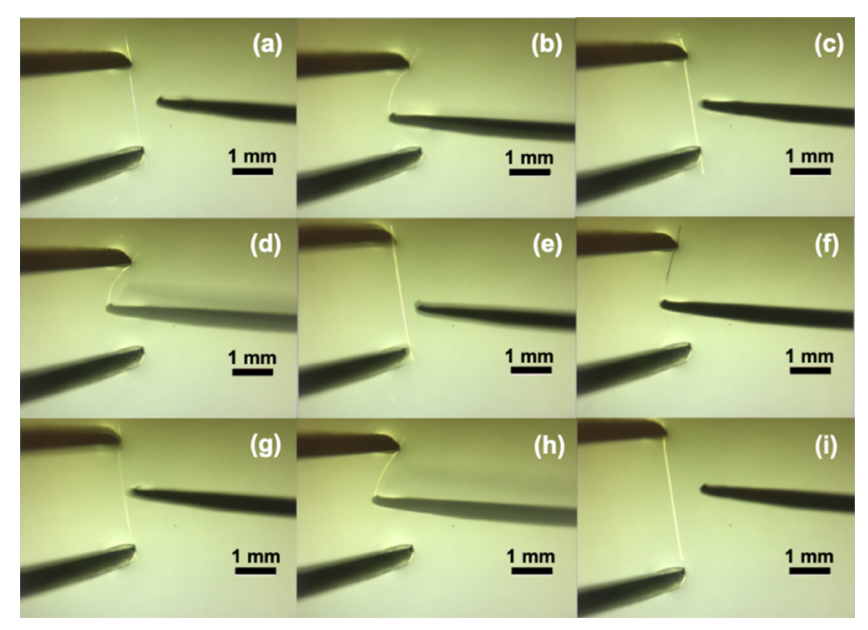

Figure 3. Screenshots of bending tests on the bendable face (001)/ $(00-1)$ of a CEL single crystal using forceps and a needle, showing four repeated cycles of bending and elastic recovery $(a-i)$.

major crystal face, $(001) /(00-1)$, by a metallic needle. An arc was formed without crystal breakage (Figure $3 \mathrm{~b}$ ), which was rapidly reversed upon withdrawal of the applied load (Figure $3 c$ ). This elastic bending phenomenon was highly reversible, as it could be performed multiple times without any sign of fatigue. However, when the load was applied to the minor face $(0-11) /(01-1)$ of the same crystal, the crystal broke before appreciable strain could be developed (see Figure S4 and Video S2). Therefore, the CEL crystal is a 1D elastic crystal because elastic deformation occurs at only one face. In addition, CEL is a rare example of a single-component elastic 
crystal without halogen bonds. Thus, unlike what was thought before, the presence of halogen bonds is not a prerequisite for elastic organic crystals. ${ }^{31}$

The CEL crystal could be elastically bent to make a closed loop (Figure 4 and Video S3). The complete shape recovery

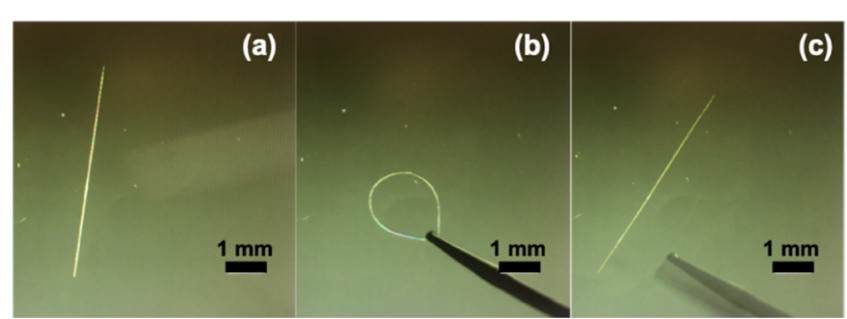

Figure 4. $(a-c)$ Looping of the elastic CEL crystal and its complete recovery.

from the loop reflects remarkable elastic flexibility. ${ }^{42}$ The elastic strain of the looped crystal, calculated using the EulerBernoulli beam-bending theory, ${ }^{43}$ is about $3.56 \%$ (see S7 for the calculation). This is significantly higher than the maximum elastic strain $(<0.5 \%)$ of most crystalline materials. ${ }^{44}$ The highest reported value of maximum elastic strain for known elastic polyhalogenated-based molecular crystals is $2 \%$ for 2,3dichlorobenzylidine-4-chloroaniline. ${ }^{25,33}$ Thus, the elastic strain of the CEL crystal is at least $78 \%$ higher than the previously known most elastically flexible organic crystal.

The mechanical properties of the bendable major face, $(001) /(00-1)$, of CEL crystals were characterized using nanoindentation (Figures 5 and S8), which provided a highly accurate measurement of the mechanical properties of molecular crystals by examining an essentially defect-free area of a sample. ${ }^{1,4,20-23,45-48}$ The elastic modulus $(E)$ and hardness $(H)$ were $16.27 \pm 0.43$ and $0.45 \pm 0.02 \mathrm{GPa}$, respectively. This $E$ value is significantly higher (28\%) than the highest $E$ reported for the elastically bendable organic crystal ( $N$-2,5-dichlorobenzylidine-4-iodo aniline). ${ }^{33}$ This is attributed to the presence of a large number of relatively weak hydrogen bonds $(\mathrm{C}-\mathrm{H} \cdots \pi, \mathrm{C}-\mathrm{H} \cdots \mathrm{F}, \mathrm{C}-\mathrm{H} \cdots \mathrm{O}$, and $\mathrm{C}-\mathrm{H} \cdots \mathrm{N})$ between CEL dimers, which are fortified by strong $\mathrm{N}-\mathrm{H} \cdots \mathrm{N}$ and $\mathrm{N}-$ $\mathrm{H}$... O hydrogen bonds, in the CEL crystal compared to those in N-2,5-dichlorobenzylidine-4-iodo aniline, where only weak hydrogen bonds $(\mathrm{C}-\mathrm{H} \cdots \mathrm{Cl})$ and halogen bonds (type $1 \mathrm{Cl} \cdots \mathrm{I}$ ) are present. Thus, incorporating both strong and weak intermolecular interactions in the crystal structure may be an effective strategy for designing highly elastic crystals with high stiffness.

All proposed qualitative mechanistic models predict inhomogeneous molecular distribution in the lattice of bent elastic crystals. $^{24,25}$ The splaying of molecules and sliding of the molecular layers in the elastically deformed crystal affect the intermolecular interactions in the three domains, that is, outer, inner, and central arcs (Figure 6). ${ }^{49}$ In the outer arc, the pronounced weakening of the intermolecular interactions is expected because of the larger intermolecular separations due to tensile strain. However, intermolecular interactions are stronger in the inner arc due to the closer proximity among molecules resulting from the compressive strain, which may even introduce new intermolecular interactions. The structural arrangement in the neutral axis (middle part) of the bent crystal is the same as the straight crystal. Such inhomogeneous interactions in a bent crystal can be experimentally

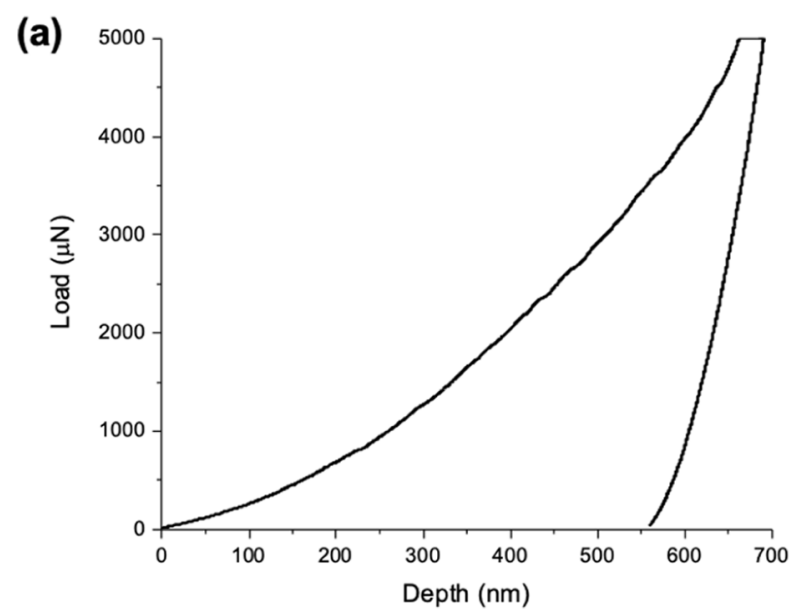

(b)

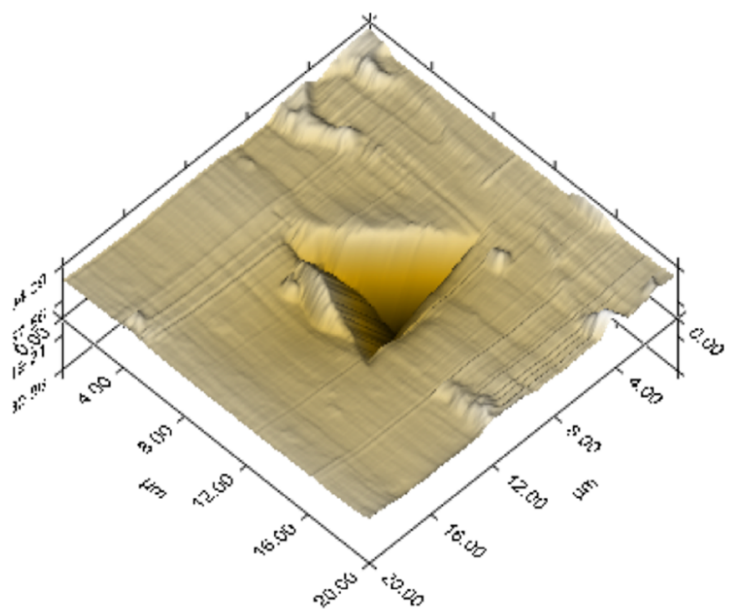

Figure 5. Nanoindentation characterization of the CEL crystal. (a) Force-depth curve and (b) 3D scan of the indented area.

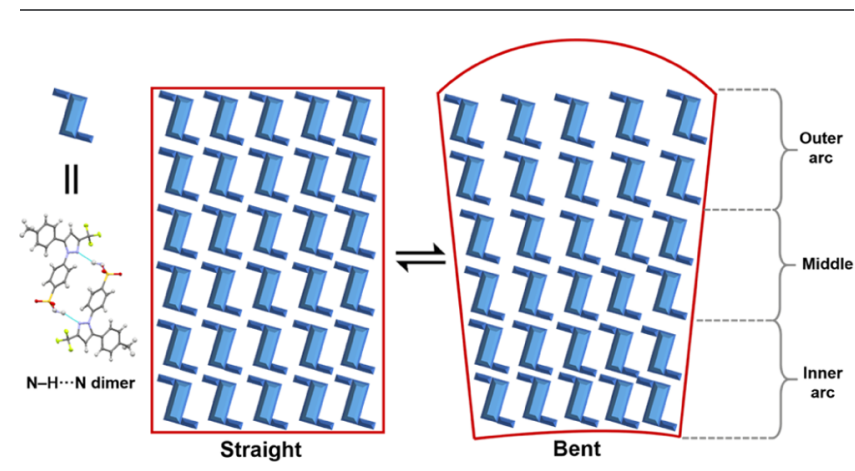

Figure 6. Schematic representation of the molecular rearrangement in the elastic crystal during the bending state.

characterized by in situ micro-Raman spectroscopy, which can measure the structural inhomogeneity in the elastically bent CEL crystal (S9 and Figure S7). ${ }^{49}$

Careful inspection of the Raman spectra in the three domains reveals broadening and shifting in bending modes of lattice vibration and aromatic $\mathrm{C}-\mathrm{H}$ bonds. ${ }^{50}$ Thus, the crucial role of aromatic $\mathrm{C}-\mathrm{H}$ bonds in the elasticity of the CEL crystal (Figure 2) is confirmed. The $102.84 \mathrm{~cm}^{-1}$ band (Figure $7 \mathrm{a}$ ) of a straight crystal corresponds to the strong lattice vibrations of CEL. Therefore, the changes in the peak indicate the prominence of the strong lattice vibration in strained domains. Strengthening the intermolecular interactions and the for- 
(a)

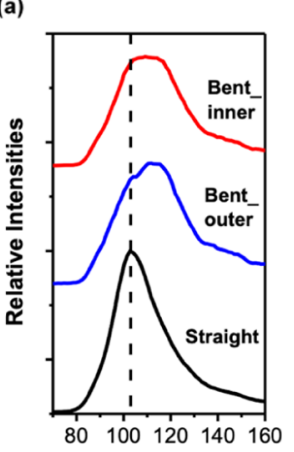

Wavenumbers $\left(\mathrm{cm}^{-1}\right)$

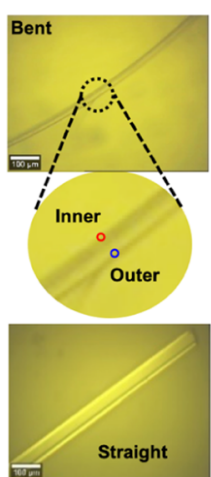

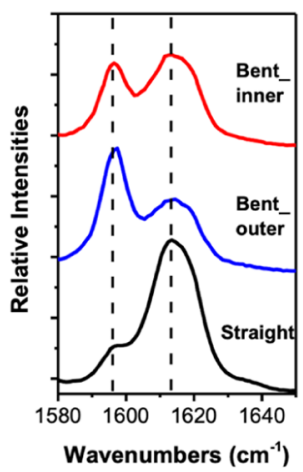

Figure 7. Raman spectra corresponding to the bent and straight crystals of CEL with red and blue circles indicating the point of data collection (inner arc and outer arc, respectively) (a) lattice vibration, and (b) aromatic symmetric in-plane bending $\delta(\mathrm{C}-\mathrm{H})$ modes of inner and outer arc of a bent CEL single crystal, as well as in the nonstrained straight state.

mation of multiple, new hydrogen bonds due to closer proximity of molecules in the compressed inner arc result in a significant broadening of the $102.84 \mathrm{~cm}^{-1}$ band. However, the same $102.84 \mathrm{~cm}^{-1}$ band in the outer arc shows a blue shift with a broadening of up to $113 \mathrm{~cm}^{-1}$ because of the fewer and weaker intermolecular interactions as the molecules are farther apart. The aromatic $\mathrm{C}-\mathrm{H}$ group of 4-methylphenyl and the pyrazol ring of CEL are involved in the formation of several weak interactions, such as $\mathrm{C}-\mathrm{H} \cdots \mathrm{F}, \mathrm{C}-\mathrm{H} \cdots \mathrm{O}$, and $\mathrm{C}-\mathrm{H} \cdots \mathrm{N}$, which act as a structural buffer during elastic bending.

The bands at 1596 and $1613 \mathrm{~cm}^{-1}$ of the unbent crystal correspond to the symmetric in-plane bending $\delta(\mathrm{C}-\mathrm{H})$ modes of the aromatic $\mathrm{C}-\mathrm{H}$ group (Figure $7 \mathrm{~b}$ ). The $1596 \mathrm{~cm}^{-1}$ band shows broadening with increased intensity in the compressed inner arc due to the formation of multiple hydrogen bonds involving the aromatic $\mathrm{C}-\mathrm{H}$ groups. However, the same band in the outer arc became sharper and blue-shifted because of weakening of the intermolecular interactions as well as strengthening of the $\mathrm{C}-\mathrm{H}$ bonds. The band at $1613 \mathrm{~cm}^{-1}$ shows significant broadening in the compressed inner arc, which is expected due to strengthening of the interactions involving the aromatic $\mathrm{C}-\mathrm{H}$ group. The intensity of the 1613 $\mathrm{cm}^{-1}$ band is diminished with broadening in the outer arc. This effect is expected for structural modifications due to induced maximum strain and, hence, longer hydrogen bonds present in the outer arc. These observations validate the mechanistic bending model of the elastic CEL crystal shown in Figure 6 and confirm the inhomogeneous spatial separations of molecules during elastic bending.

In summary, we report the first elastically bendable singlecomponent pharmaceutical crystal. Two major structural features responsible for the superior elasticity in CEL are (1) corrugated structure with criss-cross interlocked packing without slip planes and (2) numerous weak and a few strong dispersive interactions in orthogonal directions. An interlocked packing without slip planes restricts long-range molecular movement away from the equilibrium positions during elastic bending. Furthermore, the bending and nanoindentation experiments revealed a new strategy for designing highly elastic crystals by incorporating both strong and weak intermolecular interactions. The structural perturbation model was validated by a Raman spectroscopic analysis,

which confirmed the inhomogeneous spatial separation of molecules corresponding to the expected changes in distances among molecules in different domains of the bent crystal. A structural understanding of the mechanical elasticity in this single-component pharmaceutical crystal can guide future designs of molecules for mechanopharmaceutical applications, including low-cost flexible smart functional microdevices for biomedical applications with advantageous mechanical properties. $^{51}$

\section{ASSOCIATED CONTENT}

\section{S Supporting Information}

The Supporting Information is available free of charge on the ACS Publications website at DOI: 10.1021/acs.chemmater.9b00040.

Crystallographic data of CEL (CIF)

Elastic bending (MPG)

Looping and recovery (MPG)

Bending on the minor face (MPG)

Experimental details, SCXRD experiment, face indexing, 3D representation of CEL molecular conformation, corrugated layer structure, anisotropic elastic-brittle behavior, three-point bending and looping tests, nanoindentation, and Raman spectra of CEL (PDF)

\section{AUTHOR INFORMATION}

\section{Corresponding Author}

*E-mail: sunx0053@umn.edu.

ORCID ${ }^{\circ}$

Kunlin Wang: 0000-0003-1382-4933

Manish Kumar Mishra: 0000-0002-8193-3499

Changquan Calvin Sun: 0000-0001-7284-5334

\section{Author Contributions}

${ }^{\dagger}$ K.W. and M.K.M. contributed equally to this work.

\section{Notes}

The authors declare no competing financial interest.

\section{ACKNOWLEDGMENTS}

The authors acknowledge the Minnesota Supercomputing Institute (MSI) at the University of Minnesota for providing resources that contributed to the research results reported within this paper. URL: http://www.msi.umn.edu. Parts of this work were carried out in the Characterization Facility, University of Minnesota, which receives partial support from NSF through the MRSEC program. K.W. also thanks the Edward G. Rippie Fellowship and David Grant Fellowship of the University of Minnesota Department of Pharmaceutics for partial financial support.

\section{ABBREVIATION}

CEL, celecoxib

\section{REFERENCES}

(1) Mishra, M. K.; Ramamurty, U.; Desiraju, G. R. Mechanical Property Design of Molecular Solids. Curr. Opin. Solid State Mater. Sci. 2016, 20, 361-370.

(2) Saha, S.; Desiraju, G. R. Crystal Engineering of Hand-Twisted Helical Crystals. J. Am. Chem. Soc. 2017, 139, 1975-1983.

(3) Reddy, C. M.; Rama Krishna, G.; Ghosh, S. Mechanical Properties of Molecular Crystals-Applications to Crystal Engineering. CrystEngComm 2010, 12, 2296-2314. 
(4) Saha, S.; Mishra, M. K.; Reddy, C. M.; Desiraju, G. R. From Molecules to Interactions to Crystal Engineering: Mechanical Properties of Organic Solids. Acc. Chem. Res. 2018, 51, 2957-2967.

(5) Naumov, P.; Chizhik, S.; Panda, M. K.; Nath, N. K.; Boldyreva, E. Mechanically Responsive Molecular Crystals. Chem. Rev. 2015, $115,12440-12490$.

(6) Sun, C. C. Materials Science Tetrahedron-a Useful Tool for Pharmaceutical Research and Development. J. Pharm. Sci. 2009, 98, 1671-1687.

(7) Garcia-Garibay, M. A. Molecular Crystals on the Move: From Single-Crystal-to-Single-Crystal Photoreactions to Molecular Machinery. Angew. Chem., Int. Ed. 2007, 46, 8945-8947.

(8) Fratzl, P.; Barth, F. G. Biomaterial Systems for Mechanosensing and Actuation. Nature 2009, 462, 442-448.

(9) Cao, Y.; Li, H. Engineered Elastomeric Proteins with Dual Elasticity Can Be Controlled by a Molecular Regulator. Nat. Nanotechnol. 2008, 3, 512-516.

(10) Keten, S.; Xu, Z.; Ihle, B.; Buehler, M. J. Nanoconfinement Controls Stiffness, Strength and Mechanical Toughness of Beta-Sheet Crystals in Silk. Nat. Mater. 2010, 9, 359-367.

(11) Bronstein, H.; Chen, Z.; Ashraf, R. S.; Zhang, W.; Du, J.; Durrant, J. R.; Shakya Tuladhar, P.; Song, K.; Watkins, S. E.; Geerts, Y.; Wienk, M. M.; Janssen, R. A. J.; Anthopoulos, T.; Sirringhaus, H.; Heeney, M.; McCulloch, I. Thieno[3,2-B]Thiophene-Diketopyrrolopyrrole-Containing Polymers for High-Performance Organic FieldEffect Transistors and Organic Photovoltaic Devices. J. Am. Chem. Soc. 2011, 133, 3272-3275.

(12) Schmidt-Mende, L.; Fechtenkotter, A.; Mullen, K.; Moons, E.; Friend, R. H.; MacKenzie, J. D. Self-Organized Discotic Liquid Crystals for High-Efficiency Organic Photovoltaics. Science 2001, 293, $1119-1122$.

(13) Terao, F.; Morimoto, M.; Irie, M. Light-Driven MolecularCrystal Actuators: Rapid and Reversible Bending of Rodlike Mixed Crystals of Diarylethene Derivatives. Angew. Chem., Int. Ed. 2012, 51, 901-904.

(14) Worthy, A.; Grosjean, A.; Pfrunder, M. C.; Xu, Y.; Yan, C.; Edwards, G.; Clegg, J. K.; McMurtrie, J. C. Atomic Resolution of Structural Changes in Elastic Crystals of Copper(Ii) Acetylacetonate. Nat. Chem. 2018, 10, 65-69.

(15) Brock, A. J.; Whittaker, J. J.; Powell, J. A.; Pfrunder, M. C.; Grosjean, A.; Parsons, S.; McMurtrie, J. C.; Clegg, J. K. Elastically Flexible Crystals Have Disparate Mechanisms of Molecular Movement Induced by Strain and Heat. Angew. Chem., Int. Ed. 2018, 57, $11325-11328$.

(16) Desiraju, G. R. Crystal Engineering: From Molecule to Crystal. J. Am. Chem. Soc. 2013, 135, 9952-9967.

(17) Babu, N. J.; Nangia, A. Solubility Advantage of Amorphous Drugs and Pharmaceutical Cocrystals. Cryst. Growth Des. 2011, 11, $2662-2679$.

(18) Sun, C. C. Decoding Powder Tabletability: Roles of Particle Adhesion and Plasticity. J. Adhes. Sci. Technol. 2011, 25, 483-499.

(19) Smith, A. J.; Kavuru, P.; Wojtas, L.; Zaworotko, M. J.; Shytle, R. D. Cocrystals of Quercetin with Improved Solubility and Oral Bioavailability. Mol. Pharm. 2011, 8, 1867-1876.

(20) Wang, C.; Paul, S.; Wang, K.; Hu, S.; Sun, C. C. Relationships among Crystal Structures, Mechanical Properties, and Tableting Performance Probed Using Four Salts of Diphenhydramine. Cryst. Growth Des. 2017, 17, 6030-6040.

(21) Mishra, M. K.; Ramamurty, U.; Desiraju, G. R. Solid Solution Hardening of Molecular Crystals: Tautomeric Polymorphs of Omeprazole. J. Am. Chem. Soc. 2015, 137, 1794-1797.

(22) Mishra, M. K.; Desiraju, G. R.; Ramamurty, U.; Bond, A. D. Studying Microstructure in Molecular Crystals with Nanoindentation: Intergrowth Polymorphism in Felodipine. Angew. Chem., Int. Ed. 2014, 53, 13102-13105.

(23) Sanphui, P.; Mishra, M. K.; Ramamurty, U.; Desiraju, G. R. Tuning Mechanical Properties of Pharmaceutical Crystals with Multicomponent Crystals: Voriconazole as a Case Study. Mol. Pharm. 2015, 12, 889-897.
(24) Ghosh, S.; Reddy, C. M. Elastic and Bendable Caffeine Cocrystals: Implications for the Design of Flexible Organic Materials. Angew. Chem., Int. Ed. 2012, 51, 10319-10323.

(25) Ghosh, S.; Mishra, M. K.; Kadambi, S. B.; Ramamurty, U.; Desiraju, G. R. Designing Elastic Organic Crystals: Highly Flexible Polyhalogenated N-Benzylideneanilines. Angew. Chem., Int. Ed. 2015, 54, 2674-2678.

(26) Saha, S.; Desiraju, G. R. Using Structural Modularity in Cocrystals to Engineer Properties: Elasticity. Chem. Commun. 2016, 52, 7676-7679.

(27) Mukherjee, A.; Desiraju, G. R. Halogen Bonds in Some Dihalogenated Phenols: Applications to Crystal Engineering. IUCrJ 2014, 1, 49-60.

(28) Takamizawa, S.; Miyamoto, Y. Superelastic Organic Crystals. Angew. Chem., Int. Ed. 2014, 53, 6970-6973.

(29) Ghosh, S.; Mishra, M. K.; Ganguly, S.; Desiraju, G. R. Dual Stress and Thermally Driven Mechanical Properties of the Same Organic Crystal: 2,6-Dichlorobenzylidene-4-Fluoro-3-Nitroaniline. J. Am. Chem. Soc. 2015, 137, 9912-9921.

(30) Ahmed, E.; Karothu, D. P.; Naumov, P. Crystal Adaptronics: Mechanically Reconfigurable Elastic and Superelastic Molecular Crystals. Angew. Chem., Int. Ed. 2018, 57, 8837-8846.

(31) Saini, A. K.; Natarajan, K.; Mobin, S. M. A New Multitalented Azine Ligand: Elastic Bending, Single-Crystal-to-Single-Crystal Transformation and a Fluorescence Turn-on $\mathrm{Al}(\mathrm{III})$ Sensor. Chem. Commun. 2017, 53, 9870-9873.

(32) Mir, S. H.; Takasaki, Y.; Engel, E. R.; Takamizawa, S. Ferroelasticity in an Organic Crystal: A Macroscopic and Molecular Level Study. Angew. Chem., Int. Ed. 2017, 56, 15882-15885.

(33) Mishra, M. K.; Kadambi, S. B.; Ramamurty, U.; Ghosh, S. Elastic Flexibility Tuning Via Interaction Factor Modulation in Molecular Crystals. Chem. Commun. 2018, 54, 9047-9050.

(34) Keswani, R. K.; Yoon, G. S.; Sud, S.; Stringer, K. A.; Rosania, G. R. A Far-Red Fluorescent Probe for Flow Cytometry and ImageBased Functional Studies of Xenobiotic Sequestering Macrophages. Cytometry, Part A 2015, 87, 855-867.

(35) Hayashi, S.; Asano, A.; Kamiya, N.; Yokomori, Y.; Maeda, T.; Koizumi, T. Fluorescent Organic Single Crystals with Elastic Bending Flexibility: 1,4-Bis(Thien-2-Yl)-2,3,5,6-Tetrafluorobenzene Derivatives. Sci. Rep. 2017, 7, 9453.

(36) Sitti, M.; Ceylan, H.; Hu, W.; Giltinan, J.; Turan, M.; Yim, S.; Diller, E. Biomedical Applications of Untethered Mobile Milli/ Microrobots. Proc. IEEE 2015, 103, 205-224.

(37) Horstman, E. M.; Keswani, R. K.; Frey, B. A.; Rzeczycki, P. M.; LaLone, V.; Bertke, J. A.; Kenis, P. J. A.; Rosania, G. R. Elasticity in Macrophage-Synthesized Biocrystals. Angew. Chem., Int. Ed. 2017, 56, 1815-1819.

(38) Gupta, P.; Karothu, D. P.; Ahmed, E.; Naumov, P.; Nath, N. K. Thermally Twistable, Photobendable, Elastically Deformable, and Self-Healable Soft Crystals. Angew. Chem., Int. Ed. 2018, 57, 84988502.

(39) Dev, R. V.; Shashi Rekha, K.; Vyas, K.; Mohanti, S. B.; Rajender Kumar, P.; Om Reddy, G. Celecoxib, a Cox-Ii Inhibitor. Acta Crystallogr., Sect. C: Cryst. Struct. Commun. 1999, 55, IUC9900161.

(40) Modi, S. R.; Dantuluri, A. K. R.; Puri, V.; Pawar, Y. B.; Nandekar, P.; Sangamwar, A. T.; Perumalla, S. R.; Sun, C. C.; Bansal, A. K. Impact of Crystal Habit on Biopharmaceutical Performance of Celecoxib. Cryst. Growth Des. 2013, 13, 2824-2832.

(41) Face Indexing of Cel Crystal Matched with the Reported Face Indexation Data [9a] except One Minor Long Face $(01-1) /(0-11)$. This Is Not Surprising since Minor Faces of a Crystal Can Vary under Different Crystallization Environments.

(42) Bauchau, O. A.; Craig, J. I. Structural Analysis; Springer: Dordrecht, 2009; pp 173-221.

(43) Timoshenko, S. P. History of Strength of Materials; McGrawHill: New York, 1953.

(44) Telford, M. The Case for Bulk Metallic Glass. Mater. Today 2004, 7, 36-43. 
(45) Varughese, S.; Kiran, M. S. R. N.; Ramamurty, U.; Desiraju, G. R. Nanoindentation in Crystal Engineering: Quantifying Mechanical Properties of Molecular Crystals. Angew. Chem., Int. Ed. 2013, 52, 2701-2712.

(46) Mishra, M. K.; Varughese, S.; Ramamurty, U.; Desiraju, G. R. Odd-Even Effect in the Elastic Modulii of Alpha,Omega-Alkanedicarboxylic Acids. J. Am. Chem. Soc. 2013, 135, 8121-8124.

(47) Krishna, G. R.; Shi, L.; Bag, P. P.; Sun, C. C.; Reddy, C. M. Correlation among Crystal Structure, Mechanical Behavior, and Tabletability in the Co-Crystals of Vanillin Isomers. Cryst. Growth Des. 2015, 15, 1827-1832.

(48) Liu, F.; Hooks, D. E.; Li, N.; Mara, N. A.; Swift, J. A. Mechanical Properties of Anhydrous and Hydrated Uric Acid Crystals. Chem. Mater. 2018, 30, 3798-3805.

(49) Mishra, M. K.; Mishra, K.; Syed Asif, S. A.; Manimunda, P. Structural Analysis of Elastically Bent Organic Crystals Using in Situ Indentation and Micro-Raman Spectroscopy. Chem. Commun. 2017, 53, 13035-13038.

(50) Smith, G. D. E. Modern Raman Spectroscopy-a Practical Approach; John Wiley \& Sons, Ltd: West Sussex, England, 2005.

(51) Cianchetti, M.; Laschi, C.; Menciassi, A.; Dario, P. Biomedical Applications of Soft Robotics. Nat. Rev. Mater. 2018, 3, 143. 